\title{
Minimizing Energy Consumption in IR-UWB Based Wireless Sensor Networks
}

\author{
Tianqi Wang, Wendi Heinzelman and Alireza Seyedi \\ Department of Electrical and Computer Engineering, University of Rochester \\ Email: \{tiwang,wheinzel,alireza\} @ece.rochester.edu
}

\begin{abstract}
Impulse Radio Ultra-wideband (IR-UWB) communication has proven an important technique for supporting highrate, short-range, low-power communication. These are necessary criteria for emerging sensor networks, which oftentimes have very short distance communication requirements, necessitate low power operation and may require high data rates (e.g., for supporting the transmission of images or video). In this paper, using detailed models of typical IR-UWB transmitter/receiver structures, we minimize the energy consumption per information bit in a single link of an IR-UWB system, considering packet retransmissions and overhead. This minimization is realized by finding the optimum packet length and the optimum number of RAKE fingers at the receiver for different transmission distances, using differential BPSK (DBPSK) and OOK with coherent and non-coherent detection. Our results show that at very short distances, it is optimum to use OOK with non-coherent detection and large packets, and at longer distances, it is optimum to use DBPSK with coherent detection and small packets.
\end{abstract}

\section{INTRODUCTION}

Wireless sensor networks (WSNs) have been used for many applications, including environmental monitoring, health monitoring, security and surveillance [1]. These different applications for WSNs have vastly different bandwidth requirements. Take, for example, visual sensor networks (VSNs) for surveillance or health monitoring. These networks require a relatively large bandwidth to transmit/receive images and/or video in a timely manner. Recently, impulse radio ultra-wideband (IRUWB) communications has been regarded as an attractive solution to provide such high bandwidth, especially for shortrange WSN applications [2].

Energy consumption is a very important design consideration in WSNs, including IR-UWB based WSNs. Unlike in traditional communications systems, where transmit power can be flexibly adjusted to minimize the energy consumption [3] [4], there is a strict limit on the effective isotropic radiated power (EIRP) in UWB systems due to their overlay nature. Regulations mandate that the spectrum of the signal be limited to $-41.25 \mathrm{dBmW} / \mathrm{MHz}$ [2]. Due to this strict constraint on the transmit power, the traditional adaptive modulation optimization, which is mainly achieved by adjusting the transmit power, cannot be used for IR-UWB systems. However, there are other parameters of the IR-UWB system that can be adjusted.

In IR-UWB communications, the channel delays are often resolvable due to the narrow width of the IR-UWB pulse.

This work was supported in part by the National Science Foundation under grant \# ECS-0428157 and in part by a Young Investigator grant from the Office of Naval Research, \# N00014-05-1-0626.
Therefore, a RAKE receiver structure can achieve considerable diversity gain [5][6]. The diversity gain will be increased by adding more RAKE fingers, which in turn will increase the power consumption of the receiver. Therefore, the tradeoff between the diversity gain and power consumption at the receiver must be evaluated.

Packet length is another important factor that influences the energy consumption of a communication link. A long packet will increase the packet error probability; thereby increasing the average number of transmissions in an automatic-repeatrequest (ARQ) system. On the other hand, a short packet will lower the system efficiency due to the packet overhead. Thus, an optimum packet length should be found to minimize the energy consumption.

In this paper, we provide detailed power consumption models of a typical IR-UWB transmitter and both coherent and noncoherent receivers. The optimization model considers detailed power consumption models, packet structure, the ARQ procedure, and no peak power constraint. Using this model we optimize packet length and the number of RAKE fingers at different transmission distances for OOK and DBPSK, with both coherent (CO) and Non-Coherent (NC) detection.

\section{System And Channel Models}

We consider an IR-UWB system with a convolutional code. The receiver utilizes a soft-decision Viterbi decoder. The coding rate $R_{c}=\frac{1}{2}$, the constraint length $K=7$, and the coding gain $G_{c}=5.1 \mathrm{~dB}$ [7]. Moreover, no intersymbol interference (ISI) should occur, which limits the maximum data rate. Also, perfect knowledge of the wireless channel is assumed at the receiver.

\section{A. IR-UWB Transceiver Power Consumption Model}

A typical IR-UWB transmitter is shown in Figure 1. When DBPSK and OOK are used in the transmitter, the power consumption of the transmitter can be modeled as

$$
P_{t}=P_{S Y N}+E_{p} R_{p},
$$

where $E_{p}$ is the fixed energy per pulse and $R_{p}$ is the pulse rate. $R_{p}=\rho_{t} R_{b}$, where $\rho_{t}=1$ for DBPSK and $\rho_{t}=\frac{1}{2}$ for OOK, $R_{b}$ is the bit rate and we have assumed that an information bit may be 0 or 1 with equal probability. $P_{S Y N}$ represents the power consumption of the transmitter components that are independent from the data transmission, namely the clock generator and synchronizer. 


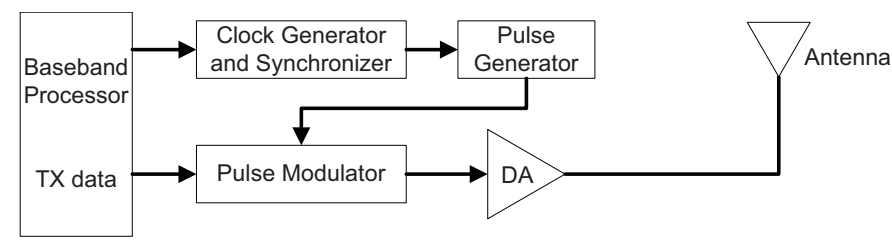

Fig. 1. The transmitter structure in an IR-UWB system.

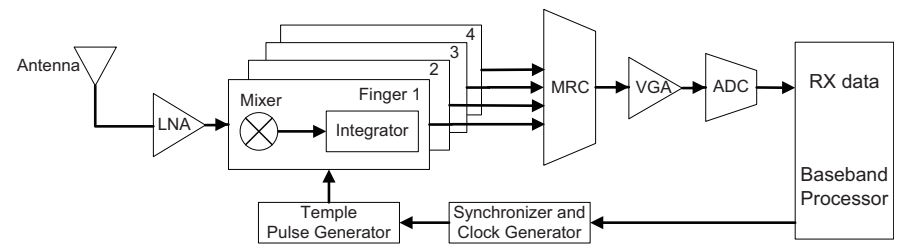

Fig. 2. A typical coherent receiver structure in IR-UWB system.

Figure 2 shows a block diagram of a typical IR-UWB receiver with four RAKE fingers and maximal ratio combining (MRC). The power consumption of a typical IR-UWB receiver can be modeled as

$$
\begin{aligned}
P_{r} & =M P_{C O R}+P_{A D C}+P_{L N A}+P_{V G A} \\
& +\rho_{r}\left(P_{G E N}+P_{S Y N}\right),
\end{aligned}
$$

where $P_{C O R}, P_{A D C}, P_{L N A}, P_{V G A}, P_{G E N}$, and $P_{S Y N}$ are the power consumptions of one correlator branch (mixer and integrator), the analog-to-digital converter, the low noise amplifier (LNA), the variable gain amplifier (VGA), the pulse generator, and clock generator and synchronizer, respectively, and $M$ represents the number of RAKE fingers at the receiver. $\rho_{r}$ is determined by the receiver structure, that is $\rho_{r}=1$ for coherent demodulation and $\rho_{r}=0$ for noncoherent demodulation, since for a noncoherent UWB receiver, the pulse generator, clock generator and synchronizer are not necessary.

\section{B. Channel Model}

1) Path Loss Model: We model the path loss in $\mathrm{dB}$ as [8]

$$
G(d)=\left\{\begin{array}{rr}
30+20.4 \log _{10} d & d \leq 11 \mathrm{~m}, \\
30+74 \log _{10} d-56 & d>11 \mathrm{~m},
\end{array}\right.
$$

2) Frequency Selective Fading Channel: In an IR-UWB system, the transmitted signal inevitably encounters frequency selective fading. The baseband channel impulse response of a frequency selective fading channel can be represented as

$$
c(\tau)=\sum_{n} \alpha_{n} e^{-\theta_{n}} \delta\left[\tau-\tau_{n}\right] .
$$

where $\alpha_{n}$ and $\theta_{n}$ have Rayleigh and uniform (over $[0,2 \pi]$ ) distributions, respectively. Furthermore, we assume an exponential power-delay profile with parameter $\gamma_{h}=39.8 \mathrm{~ns}$ [1].

\section{OPTIMIZATION MODEL}

\section{A. Effective Average Energy Consumption Per Information Bit}

The packet structure (Figure 3) consists of three components: synchronization preamble (SP), PHY-header (PHR), and payload. The energy consumption to transmit a packet once is

\begin{tabular}{|c|c|c|c|c|c|c|c|c|c|c|c|}
\hline \multicolumn{2}{|c|}{ Transmitter } & \multicolumn{4}{|c|}{$\begin{array}{l}\text { unsuccessful transmissions } \\
(m-1) \text { repetitions }\end{array}$} & \multicolumn{4}{|c|}{ successful transmission } & \multicolumn{2}{|c|}{ | } \\
\hline OFF & $T_{t r}$ & $T_{\text {on }}$ & $T_{I P S}$ & $T_{A C K}$ & $T_{I P S}$ & $T_{o n}$ & $T_{I P S}$ & $T_{A C K}$ & $T_{I P S}$ & $T_{t r}$ & OFF \\
\hline \multicolumn{2}{|c|}{$E_{t r}^{(T X)}$} & \multicolumn{2}{|c|}{$E^{(T X)} E_{I P S}^{(T X)}$} & \multicolumn{2}{|c|}{$E_{L N} E_{I P S}^{(T X)}$} & $E^{(T X)}$ & \multicolumn{3}{|c|}{$E_{I P S}^{(T X)} E_{A C K}^{(R X)} E_{I P S}^{(T X)}$} & \multicolumn{2}{|c|}{$E_{t r}^{(T X)}$} \\
\hline OFF & $T_{t r}$ & $T_{\text {on }}$ & $T_{I P S}$ & $T_{A C K}$ & $T_{I P S}$ & $T_{\text {on }}$ & $T_{I P S}$ & $T_{A C K}$ & $T_{I P S}$ & $T_{t r}$ & OFF \\
\hline
\end{tabular}
the summation of two parts: $E_{O}$, the energy consumption on

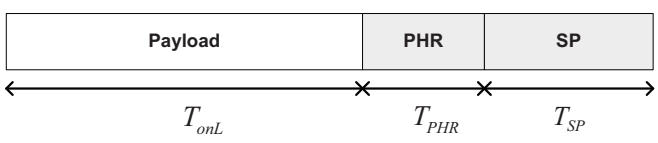

Fig. 3. Data packet structure.

Fig. 4. The transmission and reception of one packet using $m$ total transmissions.

delivering the SP and PHR, and $E_{L}$, the energy consumed on the payload.

We assume that the synchronization preamble has values $\{-1,1\}$ and is received coherently and PHR is modulated using DBPSK, always received coherently, and coded in the same manner as the payload. Therefore, the overhead energy consumption is

$$
\begin{aligned}
E_{O} & =E_{O}^{(T X)}+E_{O}^{(R X)} \\
& =\left(L_{S P}+L_{P H R} / R_{c}\right) E_{p}+P_{S Y N} T_{O}+P_{r x} T_{O}
\end{aligned}
$$

where $T_{O}=T_{S P}+T_{P H R}=\left(L_{S P}+\frac{L_{P H R}}{R_{c}}\right) / R_{\text {base }}, R_{\text {base }}$ is the fixed base data rate, i.e., $R_{b a s e}=1 \mathrm{Mbps}$ in this paper.

The energy consumption for the payload can be modeled as

$$
E_{L}=E_{L}^{(T X)}+E_{L}^{(R X)},
$$

where $E_{L}^{(T X)}$ and $E_{L}^{(R X)}$ represent the energy consumption to transmit/receive the payload containing $L_{L}$ information bits, respectively. $E_{L}^{(T X)}$ is

$$
E_{L}^{(T X)}=\rho_{t} E_{p} L_{L} / R_{c}+P_{S Y N} T_{o n L},
$$

where $T_{\text {on } L}=\frac{L_{L}}{R_{b} R_{c}}$, is the time duration to transmit the payload containing $L_{L}$ bits, and $R_{c}$ is the coding rate.

The energy consumption to receive $L_{L}$ information bits is

$$
\begin{aligned}
E_{L}^{(R X)} & =\rho_{t}\left(M P_{C O R}+P_{A D C}+P_{L N A}+P_{V G A}\right) T_{o n L} \\
& +\rho_{r}\left(P_{G E N}+P_{S Y N}\right) T_{o n L} .
\end{aligned}
$$

To guarantee the successful reception of one packet, an automatic repeat-request (ARQ) protocol is used. A delivery procedure involving $m-1$ retransmissions is shown in Figure 4. The inter packet space (IPS) is set as $T_{I P S}=200 \mu \mathrm{s}$. The power consumption during $T_{I P S}$ is mainly due to the clock generator and synchronizer. Therefore, the corresponding energy consumption at the transmitter is $E_{I P S}^{(T X)}=P_{S Y N} T_{I P S}$, while the receiver consumes $E_{I P S}^{(R X)}=\rho_{r} P_{S Y N} T_{I P S}$.

We assume that before transmission or reception of a packet, the transmitter and receiver spend $T_{t r}=800 \mu$ s to go from the off (sleep) state to an on (active) state. During this time period, 
the transmitter consumes $E_{t r}^{(T X)}=P_{S Y N} T_{t r}$ amount of energy to start the front end clock generator and synchronizer. Similarly, the receiver consumes $E_{t r}^{(R X)}=\rho_{r} P_{S Y N} T_{t r}$.

$T_{o n}$ is the time duration for the transmission of one packet. That is

$$
\begin{aligned}
T_{o n} & =T_{S P}+T_{P H R}+T_{o n L}, \\
& =\left(L_{S P}+\frac{L_{P H R}}{R_{c}}\right) / R_{\text {base }}+\frac{L_{L}}{R_{b} R_{c}} .
\end{aligned}
$$

The energy consumptions at the transmitter and receiver during $T_{\text {on }}$ are

$$
\begin{aligned}
& E^{(T X)}=E_{L}^{(T X)}+E_{O}^{(T X)}, \\
& E^{(R X)}=E_{L}^{(R X)}+E_{O}^{(R X)} .
\end{aligned}
$$

where $E_{L}^{(T X)}, E_{O}^{(T X)}, E_{L}^{(R X)}$ and $E_{O}^{(R X)}$ are given in (5), (6), (7) and (8), respectively.

$T_{A C K}$ is the time period when the transmitter listens for an acknowledgement. We set $T_{A C K} \approx T_{O}$. Overall, the definitions of the energy consumptions within one transmission are summarized as follows

$$
\begin{aligned}
E_{I P S} & =2 E_{I P S}^{(T X)}+2 E_{I P S}^{(R X)}, \\
E_{L N} & =\rho_{r} P_{S Y N} T_{A C K}, \\
E_{T R A N} & =2 E_{t r}^{(T X)}+2 E_{t r}^{(R X)}, \\
E_{A C K}^{(R X)} & =P_{r x} T_{A C K}, \\
E_{A C K}^{(T X)} & =\left(L_{S P}+L_{P H R} / R_{c}\right) E_{p}+P_{S Y N} T_{A C K} .
\end{aligned}
$$

Therefore, the effective average energy consumption per one successful delivery can be expressed as

$$
\begin{aligned}
E & =\left(E^{(T X)}+E^{(R X)}+E_{L N}+E_{I P S}\right) N \\
& -E_{L N}+E_{T R A N}+E_{A C K}^{(T X)}+E_{A C K},
\end{aligned}
$$

where $N$ is the average number of transmissions/receptions required to successfully deliver one packet. The average number of transmissions $N=\frac{1}{\left(1-P_{b}\right)^{L_{L}}}$, where $P_{b}$ is the average bit error probability (BEP). Note that $\left(1-P_{b}\right)^{L_{L}}$ is the probability that a packet is received correctly.

\section{B. Average Bit Error Probability Over Frequency Selective Channels}

To derive the average BEP, , first we investigate the probability density function (pdf) of the output SNR after the MRC. The instantaneous SNR at the $k^{\text {th }}$ finger is

$$
\gamma_{k}(t)=\frac{\left|\alpha_{k}(t)\right|^{2} P_{t} G_{c}}{G_{d} \sigma^{2}}
$$

where $P_{t}$ is the transmit power, $G_{c}$ is the coding gain, $G_{d}$ denotes the path loss at distance $d$, and $\sigma^{2}$ represents the noise power at the receiver. The instantaneous SNR at the output of the MRC is $\gamma(t)=\sum_{k=1}^{M} \gamma_{k}(t)$.

The pdf of $\gamma(t)$ is [7]

$$
p(\gamma)=\sum_{k=1}^{M} \frac{\pi_{k}}{\bar{\gamma}_{k}} e^{-\gamma / \bar{\gamma}_{k}},
$$

where $\bar{\gamma}_{k}=\frac{\mathbf{E}\left[\left|\alpha_{k}(t)\right|^{2}\right] P_{t} G_{c}}{G_{d} \sigma^{2}}$ is the average received SNR at the $k_{t h}$ finger, and $\pi_{k}=\prod_{i=1, i \neq k}^{M} \frac{\bar{\gamma}_{k}}{\bar{\gamma}_{k}-\bar{\gamma}_{i}}$.
TABLE I

BEPS OVER FREQUENCY-SELECTIVE CHANNELS

\begin{tabular}{|c|c|}
\hline Modulation Schemes & BEP \\
\hline DBPSK-CO & $P_{b, \text { DBPSK-CO }}=\sum_{k=1}^{M} \pi_{k}\left[1-\sqrt{\frac{\bar{\gamma}_{k}}{1+\bar{\gamma}_{k}}}\right]$ \\
\hline OOK-CO & $P_{b, \text { OOK-CO }} \approx \frac{1}{2} \sum_{k=1}^{M} \pi_{k}\left[1-\sqrt{\frac{\bar{\gamma}_{k}}{2+\bar{\gamma}_{k}}}\right]$ \\
\hline DBPSK-NC & $P_{b, \text { DBPSK-NC }}=\frac{1}{2} \sum_{k=1}^{M} \frac{\pi_{k}}{1+\bar{\gamma}_{k}}$ \\
\hline OOK-NC & $P_{b, \text { OOK-NC }} \approx \frac{1}{2} \sum_{k=1}^{M} \pi_{k}\left[1-\sqrt{\frac{m_{\gamma} \bar{\gamma}_{k}}{2+m_{\gamma} \bar{\gamma}_{k}}}\right]$ \\
\hline
\end{tabular}

The average bit error probability of DBPSK-CO can be found by averaging the BEP of DBPSK-CO over an AWGN channel, with the pdf of $\gamma(t)$ indicated by (14). We have the BEP of DBPSK-CO detection over an AWGN channel as [9]

$$
P_{b, \text { DBPSK-CO,AWGN }}=2 Q(\sqrt{2 \gamma})[1-Q(\sqrt{2 \gamma})] .
$$

The resulting average BEP can be approximated as

$$
\begin{aligned}
P_{b, \text { DBPSK-CO }} & =\int_{0}^{\infty} P_{b, \text { DBPSK-CO,AWGN }} p(\gamma) d \gamma \\
& \approx \sum_{k=1}^{M} \pi_{k}\left[1-\sqrt{\frac{\bar{\gamma}_{k}}{1+\bar{\gamma}_{k}}}\right] .
\end{aligned}
$$

The average bit error probabilities of OOK-CO, DBPSK$\mathrm{NC}$, OOK-NC can be obtained following a similar procedure as in the DBPSK-CO case. The results are summarized in Table I.

\section{Optimization Model}

The effective energy consumption per information bit is

$$
\begin{aligned}
E_{b} & =\left(E^{(T X)}+E^{(R X)}+E_{L N}+E_{I P S}\right) /\left(L_{L}\left(1-P_{b}\right)^{L_{L}}\right) \\
& +\left(E_{T R A N}+E_{A C K}^{(T X)}+E_{A C K}-E_{L N}\right) / L_{L} .
\end{aligned}
$$

Our goal is to find an optimum combination of the modulation scheme, the data rate $\left(R_{b}\right)$, the packet length $\left(L_{L}\right)$, and the number of RAKE fingers at the receiver $(M)$ over a slow frequency-selective channel for a given transmission distance, that minimize the effective energy consumption per information bit denoted by (16).

1) Optimum data rate: It is easy to see that data rate only appears in the denominator in (16). Therefore, the optimum data rate is always the highest possible data rate. That is, $R_{b} \approx$ $\frac{1}{D_{s}}=25 \mathrm{Mbps}$, where $D_{S}=40 \mathrm{~ns}$ is the maximum excess delay.

2) Optimum packet length: Removing the integer constraint on $L_{L}$, it is straight forward to find the closed-form optimum packet length by solving $\frac{\partial E_{b}}{\partial L_{L}}=0$. At high SNR, the result is

$$
L_{L}^{*} \approx \frac{-P_{b}(A+B)+\sqrt{P_{b}^{2}(A+B)^{2}+4(A+B) C P_{b}}}{2 C P_{b}},
$$

where

$$
\begin{aligned}
A & =E_{T R A N}+E_{A C K}^{(T X)}+E_{A C K}-E_{L N} \\
B & =E_{I P S}+E_{L N}+E_{O}^{(T X)}+E_{O}^{(R X)}+E_{L}^{(R X)} \\
C & =\left(\rho_{t} E_{p}+P_{S Y N} / R_{b}\right) / R_{c} \\
& +\left[\rho_{t}\left(M P_{C O R}+P_{A D C}+P_{L N A}+P_{V G A}\right)\right. \\
& \left.+\rho_{r}\left(P_{G E N}+P_{S Y N}\right)\right] /\left(R_{b} R_{c}\right) .
\end{aligned}
$$

As indicated by (17), $L_{L}^{*}$ will decrease as BEP increases. In an extreme situation where $P_{b}=0, L_{L}^{*}$ will be infinitely large. 
TABLE II

POWER CONSUMPTION PARAMETERS

\begin{tabular}{|c|c|c|c|c|c|c|}
\hline Device & $P_{S Y N}$ & $P_{A D C}$ & $P_{G E N}$ & $P_{V G A}$ & $P_{L N A}$ & $P_{C O R}$ \\
\hline Power(mW) & 30.6 & 2.2 & 2.8 & 22 & 9.4 & 10.08 \\
\hline
\end{tabular}

In applications, the packet length can always be the nearest integer of the resultant $L_{L}^{*}$.

As mentioned previously, the optimum data rate is always the maximum allowable data rate. Thus, the transmit power, as shown in (1), is also fixed at the maximum value. Therefore, the BEP at a given transmission distance for a given modulation scheme is solely determined by the number of RAKE fingers at the receiver. Since the BEP follows a non-increasing function of the number of RAKE fingers, $L_{L}^{*}$ follows a nondecreasing function of the number of RAKE fingers.

3) Optimum RAKE fingers: The optimum number of RAKE fingers reflects the tradeoff between the power consumption cost, $M P_{C O R}$ and the received power gain, $\mathbf{E}\left[\left|\alpha_{k}\right|^{2}\right] P_{t} G_{c} / G_{d}$. A numerical optimization can be performed over $M$. From numerical optimization, we find that, at short distances, a small number of RAKE fingers is enough to provide a low BEP to avoid retransmission. As transmission distance increases, the number of RAKE fingers has to increase to guarantee a low BEP, meanwhile the received power gain per RAKE finger will decrease as the path loss increases. After a certain transmission distance, the received power gain from higher numbers of RAKE fingers can no longer compensate the power consumption cost. Thus the optimum number of RAKE fingers will start to decrease.

\section{RESUlts}

We assume that $B=1.498 \mathrm{GHz}, L_{P H R}=16$ symbols, $L_{S P}=1024$ symbols [2], coding rate $R_{c}=1 / 2$, and coding gain $G_{c}=5.1 \mathrm{~dB}$. The maximum excess delay is $D_{S}=$ $40 \mathrm{~ns}$ which limits the maximum data rate to $25 \mathrm{Mbps}$. The power consumptions of the transmitter/receiver components are shown in Table II [10]-[14]. The fixed emitted energy per pulse is $E_{p}=4.5 \mathrm{pJ} /$ pulse. Therefore, the maximum amount of transmit power is $P_{t}=E_{p} R_{b}=0.113 \mathrm{~mW}$. Also, we assume $T_{I P S}=200 \mu \mathrm{s}$ and $T_{t r}=400 \mu \mathrm{s}$.

The overall minimized energy consumption per information bit is shown in Figure 5. OOK-NC consumes the least amount of energy when $d \leq 5 \mathrm{~m}$, while OOK-CO offers the lowest energy consumption per information bit when transmission distance is around 6 meters. When $7 \mathrm{~m} \leq d<9 \mathrm{~m}$, DBPSK$\mathrm{NC}$ is the most energy-efficient choice. When transmission distance increases to more than $9 \mathrm{~m}$, DBPSK-CO is the most energy efficient scheme. This trend reflects the balance between the transmitter energy consumption and the receiver energy consumption. At a short transmission distance, the less robust schemes (OOK) require less power consumption at the transmitter/receiver and provide a BEP low enough to avoid excessive retransmissions. Therefore, the OOK schemes have a high energy efficiency at short transmission distances. However, as transmission distance increases, OOK schemes

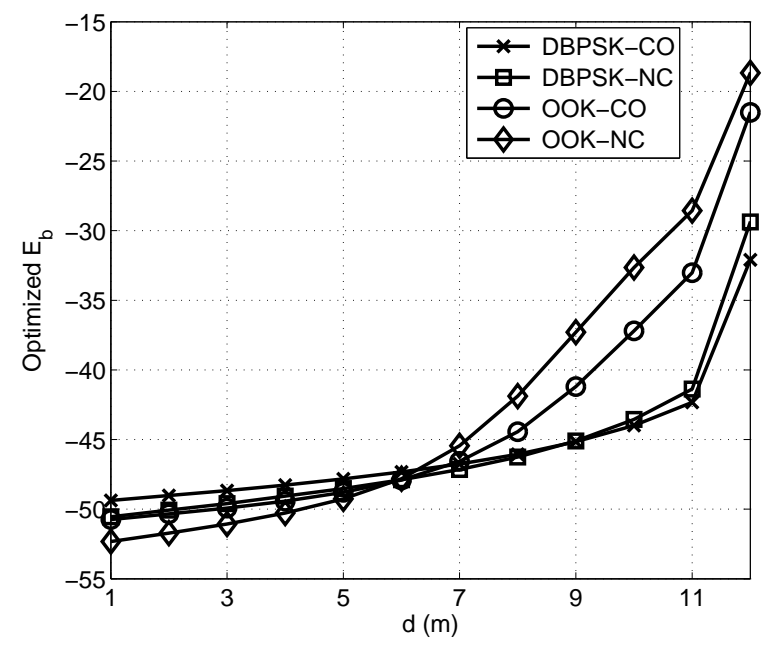

Fig. 5. The minimized energy consumption per information bit.

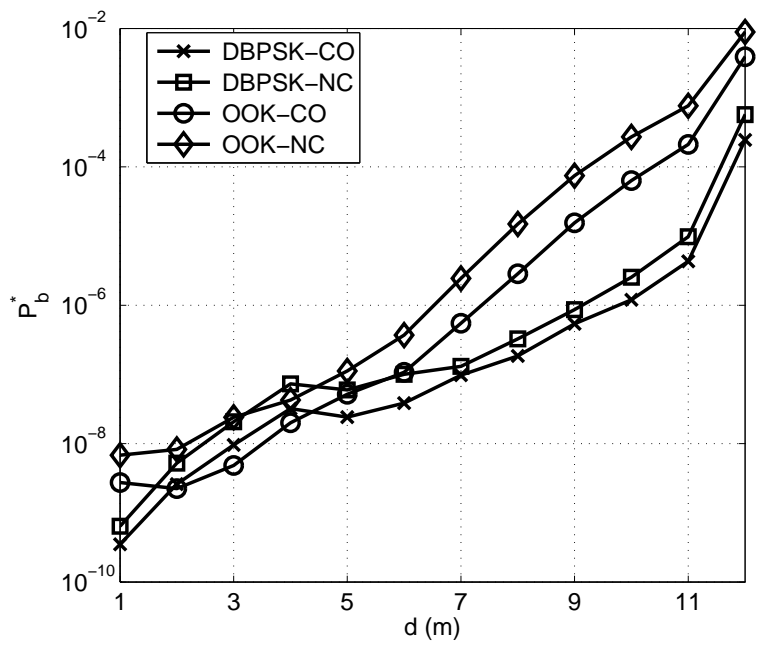

Fig. 6. The optimum target BEP versus distance.

require a large number of RAKE fingers to maintain a low BEP; thereby the receiver power consumption will increase dramatically when using OOK schemes. On the other hand, the more robust schemes (DBPSK) consume much less power at the receiver since they need fewer RAKE fingers to achieve a low BEP. Thus, as transmission distance increases, these schemes will become the energy efficient schemes.

The optimum BEPs and optimum packet lengths of different modulation schemes are shown in Figures 6 and 7, respectively. As shown in Figure 6, as the transmission distance increases, the optimum BEP will increase since it will consume increasingly more power at the receiver to maintain a low BEP as $d$ increases. Therefore, the optimum choice is to lower the target BEPs to avoid a dramatic increase in the number of RAKE fingers. Correspondingly, as shown in Figure 7, the optimum packet length will decrease as $d$ increases to avoid costly retransmissions caused by higher BEP, since a short 


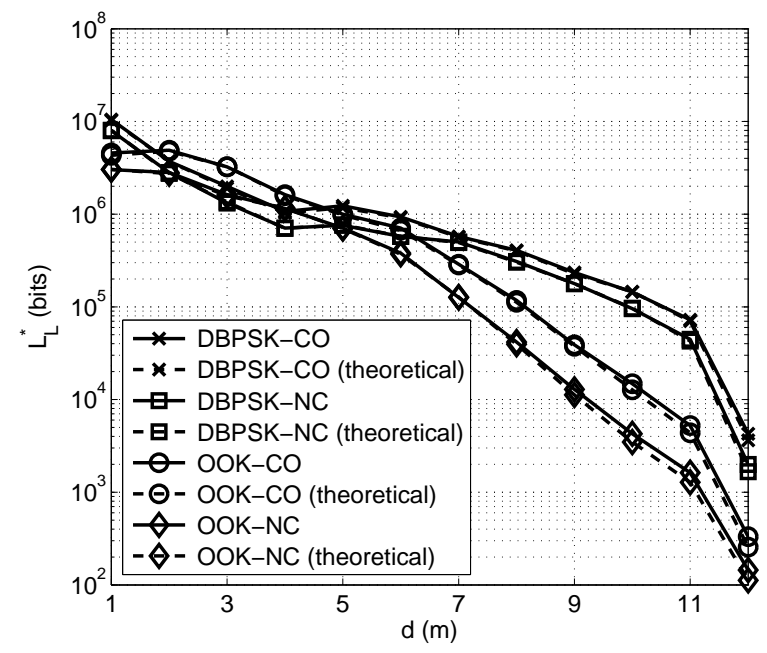

Fig. 7. The optimum packet length versus distance.

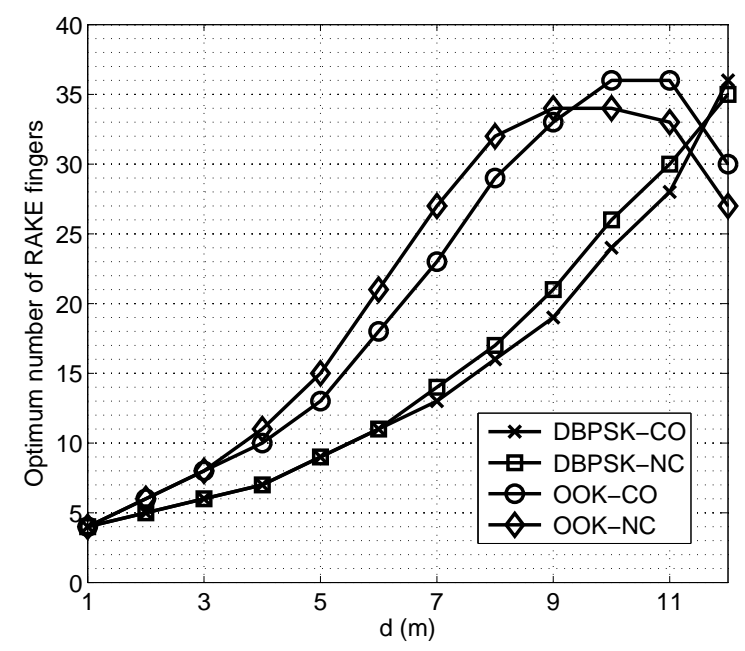

Fig. 8. The optimum number of RAKE fingers versus distance.

packet length results in a lower packet error probability. Also shown in Figure 7 are the theoretical values of $L_{L}^{*}$, which coincide well with the calculated values. Theoretical $L_{L}^{*}$ is calculated using (17) with $P_{b}=P_{b}^{*}$.

Figure 8 shows the optimum numbers of RAKE fingers at the receiver versus distance. As the transmission distance increases, the optimum numbers of RAKE fingers will increase up to a certain value, then start to decrease. This is due to the change of balance between the diversity gain and the power cost induced by each additional RAKE finger. For example, at $d=2 \mathrm{~m}$, OOK-NC with $20 \mathrm{RAKE}$ fingers at the receiver $\left(P_{r}=235.2 \mathrm{~mW}\right)$ can achieve a BEP of $2 \times 10^{-4}$, while OOK-NC with 5 RAKE fingers at the receiver $\left(P_{r}=84 \mathrm{~mW}\right)$ can only achieve a BEP of $1.5 \times 10^{-2}$. This corresponds to a $98.67 \%$ decrease in BEP at the cost of 2.8 times increase in power. However, when $d \approx 10 \mathrm{~m}$, OOK-NC with $20 \mathrm{RAKE}$ fingers at the receiver can achieve a BEP of 0.2344 while
OOK-NC with 5 RAKE fingers at the receiver can achieve a BEP of 0.3156 . This corresponds to a $25.73 \%$ decrease in BEP at the cost of 2.8 times increase in power, which indicates that the decrease in BEP cannot compensate the increase in power cost. Thus, at large transmission distances, increasing the number of RAKE fingers cannot improve the system performance in terms of energy. Note that the optimum receiver power consumptions of different modulation schemes at different transmission distances exactly follow the trend of the optimum number of RAKE fingers shown in Figure 8.

\section{CONCLUSions}

In this paper, we provided the power consumption models of typical transmitter/receiver structures that are currently used in IR-UWB systems. Then, under the assumption of a frequency selective time-invariant channel, a minimization of energy consumption per information bit considering packet overhead, retransmission, and number of RAKE fingers is found. The numerical results show that OOK-NC is energy efficient for short transmission distances, DBPSK-NC is suitable for medium transmission distances, and DBPSK-CO provides the most energy efficiency at large transmission distances. Another observation is that, as the transmission distance increases, the optimum packet length decreases. Finally, an optimum number of RAKE fingers exists under the assumption of a frequency selective time-invariant channel with an exponentiallydecaying power delay profile.

\section{REFERENCES}

[1] D. Cassioli, M. Z. Win, and A. F. Molisch, "A statistical model for the UWB indoor channel," Proc. Vehicular Technology Conference(VTC), vol. 2, pp. 1159-1163, 2001.

[2] IEEE, "IEEE P802.15.4a./d5," Sep. 2006.

[3] T. Wang, W. Heinzelman, and A. Seyedi, "Minimization of transceiver energy consumption in wireless sensor networks with awgn channels," To appear in Proc. Allerton Conference, Sep. 2008.

[4] S. Cui, A. J. Goldsmith, and A. Bahai, "Energy-contrained modulation optimization," IEEE Trans. on Wireless Communications, vol. 4, no. 8, pp. 2349-2360, Sep. 2005.

[5] M. Z. Win and R. A. Scholtz, "On the energy capture of ultrawide bandwidth signal in dense multipath environments," IEEE Communications Letters, vol. 2, no. 9, 1998.

[6] D. Cassioli, M. Z. Win, F. Vatalaro, and A. F. Molisch, "Performance of low-complexity RAKE reception in a realistic UWB channel," Proc. IEEE International Conference on Communications (ICC), 2002.

[7] J. G. Proakis, Digital Communications, 4th ed. MA: Addison-Wesley, 1972.

[8] D. Cassioli, M. Z. Win, and A. F. Molisch, "A statistical model for the UWB indoor channel,' Proc. Vehicular Technology Conference (VTC), 2001.

[9] B. Sklar, Digital Communications: Fundamentals and Applications, 2nd ed. Prentice Hall PTR, 2001.

[10] A. Medi and W. Namgoong, "A high data-rate energy-efficient interference-tolerant fully integrated CMOS frequency channelized UWB transceiver for impulse radio," Proc. JSSC, 2008.

[11] H. Kao, A. Chin, K. Chang, and S. McAlister, "A low-power currentreuse LNA for ultra-wideband wireless receivers from 3.1 to $10.6 \mathrm{GHz}$," Proc. SiRF07, 2007.

[12] Y. Gao, K. Cai, Y. Zheng, and B.-L. Ooi, “A wideband CMOS multiplier for UWB application," Proc. ICUWB, 2007.

[13] C. T. Fu and H. Luong, "A CMOS linear-in-dB high-linearity variablegain amplifier for UWB receivers," Proc. ASSCC, 2007.

[14] B. Verbruggen, J. Craninckx, M. Kuijk, P. Wambacq, and G. Van der Plas, "A $2.2 \mathrm{~mW} \mathrm{5b} 1.75 \mathrm{GS} / \mathrm{s}$ folding flash ADC in $90 \mathrm{~nm}$ digital CMOS," Proc. ISSCC, 2007. 